\title{
Hopf Bifurcation for a FitzHugh-Nagumo Model with Time Delay in a Network
}

\author{
Suxia Wang \\ School of Finance and Mathematics, Huainan Normal University, Huainan 232038, China \\ Correspondence should be addressed to Suxia Wang; wsx0227@163.com
}

Received 1 April 2021; Accepted 27 May 2021; Published 9 July 2021

Academic Editor: Ahmed Mostafa Khalil

Copyright (C) 2021 Suxia Wang. This is an open access article distributed under the Creative Commons Attribution License, which permits unrestricted use, distribution, and reproduction in any medium, provided the original work is properly cited.

\begin{abstract}
A reaction diffusion system is used to study the interaction between species in a population dynamic system. It is not only used in a population dynamic system with the diffusion phenomenon but also used in physical chemistry, medicine, and animal and plant protection. It has been studied by more and more scholars in recent years. The FitzHugh-Nagumo model is one of the most famous reaction-diffusion models. This article takes a deeper look at a FitzHugh-Nagumo model in a network with time delay. Firstly, we studied the linear stability of the equilibrium, then the existence of Hopf bifurcation is given, and finally, the stability of the Hopf bifurcation is introduced.
\end{abstract}

\section{Introduction}

Reaction-diffusion equations are widely used in ecology and biology fields [1-3]. The Hopf bifurcation is very useful in practice; in [4-7], the turing instability is analyzed. Turing [8] first introduced the coupled reaction-diffusion equations; he pointed out that the stable process could evolve into an instability with diffusive effects. As a time- and spacebreaking symmetry, Turing-Hopf bifurcation is a wellknown mechanism for generating spatiotemporal patterns $[9,10]$.

The FitzHugh-Nagumo model is one of the most famous reaction-diffusion models; Hodgkin and Huxle first introduced the FitzHugh-Nagumo model. They used the model to study electrical impulses on nerve fibers [11-13]. In the Fitzhugh-Nagumo model, the variables of equations for the voltage $r$ and $s$ have many forms, and the following form is commonly used:

$$
\left\{\begin{array}{l}
\frac{\partial r}{\partial t}=\frac{\partial^{2} r}{\partial x^{2}}-r+I+f(r) \\
\frac{\partial s}{\partial t}=a r-b s
\end{array}\right.
$$

The function $f(r)$ is positive feedback, and it is a thirdorder polynomial; however, the function $r$ is negative feedback. In [14-18], the pattern formation in the FitzHugh-Nagumo system is studied frequently. In 2001, Medvedev and Kopell [19] introduced the FitzHugh-Nagumo oscillators with coupled electrically, and the synchronization and transience are obtained; in 2004, Kostova et al. [20] investigated the revised FitzHugh-Nagumo equation. The FitzHugh-Nagumo model with time delays is considered by C. J. in 2013 [21]. Based on the abovementioned research, we discussed the FitzHugh-Nagumo model with delay as follows:

$$
\left\{\begin{array}{l}
\frac{\partial r}{\partial t}=-r^{3}(t, x)+(\alpha+1) r^{2}(t, x)-\alpha r(t, x)-s(t-\tau, x)+D_{r} \Delta r \\
\frac{\partial s}{\partial t}=a \beta r(t, x)-a s(t, x)+b D_{s} \Delta s .
\end{array}\right.
$$

In the abovementioned system, $r(t, x)$ is the membrane potential, $s(t, x)$ is the recovery variable, $D_{r}$ and $D_{s}$ are the diffusive coefficients of $r(t, x)$ and $s(t, x)$, respectively, $\alpha$ is the excitatory threshold, $b$ is the diffusion coefficient, $a$ is the excitability and $\beta, a$ are parameters, they can change the 
rest-state and dynamics, $\triangle$ denotes the Laplacian operator which describes the diffusion, and $\tau$ represents the time delay. For detail, one can see [22]. This model is based on a spatially continuous domain, that is, the entire landscape is spatially continuous; in this paper, we introduce a network that owns system (2).

$$
\left\{\begin{array}{l}
\dot{r}_{i}(t)=-r_{i}^{3}(t)+(\alpha+1) r_{i}^{2}(t)-\alpha r_{i}(t)-s_{i}(t-\tau)+D_{r} \sum_{j=1}^{n} L_{i j} r_{j}(t) \\
\dot{s}_{i}(t)=a \beta r_{i}(t)-a s_{i}(t)+D_{s} \sum_{j=1}^{n} L_{i j} s_{j}(t)
\end{array}\right.
$$

where $G=\left(g_{i j}\right)_{n \times n}$ is an adjacency matrix, whose entry $g_{i j}$ equals 1 if there exists an edge from node $j$ to node $i$ and 0 otherwise. It is obvious that $g_{i i}=0$ for $i=1,2, \ldots, n$. We assume that $G$ is strongly connected, i.e., for any pair of distinct nodes, there exists a path from one node to the other. It is known that the adjacency matrix $G$ is irreducible [23]. The definition of the Laplacian matrix $L$ is as follows:

$$
L=\left(\begin{array}{cccc}
-\sum_{k=1}^{n} g_{1 k} & g_{12} & \ldots & g_{1 n} \\
g_{21} & -\sum_{k=1}^{n} g_{2 k} & \ldots & g_{2 n} \\
\ldots & \ldots & \ldots & \ldots \\
g_{n 1} & g_{n 2} & \ldots & -\sum_{k=1}^{n} g_{n k}
\end{array}\right) .
$$

The rest of this paper is designed as follows. In Section 2, we give the linear stability of the equilibrium and the existence of Hopf bifurcation. In Section 3, the stability of the Hopf bifurcation is introduced.

\section{Stability of the Equilibrium and Hopf Bifurcation}

By simple calculation, we know that equation (3) has three equilibriums $(0,0) ;\left(\widehat{r}_{1}, \widehat{s}_{1}\right)$; and $\left(\widehat{r}_{2}, \widehat{s}_{2}\right)$, where

$$
\begin{array}{ll}
\widehat{r}_{1}=\frac{\alpha+1+\sqrt{(\alpha+1)^{2}-4 \beta}}{2}, & \widehat{s}_{1}=\beta \widehat{r}_{1}, \\
\widehat{r}_{2}=\frac{\alpha+1-\sqrt{(\alpha+1)^{2}-4 \beta}}{2}, & \widehat{s}_{2}=\beta \widehat{r}_{2} .
\end{array}
$$

The interior equilibrium exists if and only if

$$
(\alpha+1)^{2}>4 \beta .
$$

Now, we focus on the stability properties of the coexisting equilibrium $\left(\widehat{r}_{1}, \widehat{s}_{1}\right),\left(\widehat{r}_{2}, \widehat{s}_{2}\right)$, we define the small perturbations $\delta r_{i}=r_{i}-\widehat{r} ;, \delta s_{i}=s_{i}-\widehat{s}$, and system (3) can be expressed as follows:

$$
\left(\begin{array}{c}
\delta \dot{r}_{i} \\
\delta \dot{s}_{i}
\end{array}\right)=J_{1}\left(\begin{array}{c}
\delta r_{i} \\
\delta s_{i}
\end{array}\right)+J_{2}\left(\begin{array}{c}
\delta r_{i, \tau} \\
\delta s_{i, \tau}
\end{array}\right)+D\left(\begin{array}{c}
\sum_{j=1}^{n} L_{i j} \delta r_{j} \\
\sum_{j=1}^{n} L_{i j} \delta s_{j}
\end{array}\right)
$$

where $\delta r_{i, \tau}=\delta r_{i}(t-\tau)$ and

$$
\begin{aligned}
& J_{1}=\left(\begin{array}{cc}
a_{11} & -1 \\
b \beta & -b
\end{array}\right), \\
& J_{2}=\left(\begin{array}{cc}
0 & -1 \\
0 & 0
\end{array}\right), \\
& D=\left(\begin{array}{cc}
D_{r} & 0 \\
0 & D_{s}
\end{array}\right),
\end{aligned}
$$

where $a_{11}=-3 \widehat{r}^{2}+2(\alpha+1) \widehat{r}-\alpha$.

As in [24], we use $0=\Lambda^{1}>\Lambda^{2}>\cdots>\Lambda^{n}$ to express the eigenvalues of the Laplacian matrix $L$ and $\left\{\Phi^{\gamma}: \gamma=1,2, \ldots, n\right\}$ to express each eigenvector relevant to a topological eigenvalue $\Lambda^{\gamma}$, so we can get $\sum_{j=1}^{n} L_{i j} \Phi_{j}^{\gamma}=\Lambda^{\gamma} \Phi_{i}^{\gamma}$. Let $T$ be the transpose and $\left(\delta r_{i}(t), \delta s_{i}(t)\right)^{T}$ express any small disturbance in the equilibrium state; we can have the following basis decomposition:

$$
\left(\begin{array}{c}
\delta r_{i}(t) \\
\delta s_{i}(t)
\end{array}\right)=\sum_{\alpha=1}^{n}\left(\begin{array}{c}
c_{1}^{\gamma} \\
c_{2}^{\gamma}
\end{array}\right) e^{\lambda_{\gamma} t} \Phi_{\gamma}^{\alpha}, \quad(i=1,2, \ldots, n) .
$$

Inserting (9) into (7), using the orthogonality of the eigenvectors, we get

$$
\lambda_{\gamma}\left(\begin{array}{c}
c_{1}^{\gamma} \\
c_{2}^{\gamma}
\end{array}\right) e^{\lambda_{\gamma}} t=J_{1}\left(\begin{array}{c}
c_{1}^{\gamma} \\
c_{2}^{\gamma}
\end{array}\right) e^{\lambda_{\gamma} t}+J_{2}\left(\begin{array}{c}
c_{1}^{\gamma} \\
c_{2}^{\gamma}
\end{array}\right) e^{\lambda_{\gamma}(t-\tau)}+\Lambda^{\gamma} D\left(\begin{array}{c}
c_{1}^{\gamma} \\
c_{2}^{\gamma}
\end{array}\right) e^{\lambda_{\gamma} t} .
$$

So, the characteristic equation can be written as

$$
\Delta\left(\lambda_{\gamma, \tau}\right)=\operatorname{det}\left(\lambda_{\gamma} I-\left(J_{1}+e^{-\lambda_{\gamma} \tau} J_{2}+\Lambda^{\gamma} D\right)\right) .
$$

Substituting (8) into (11), the characteristic equation of (3) can be written as

$$
\lambda_{\gamma}^{2}-\left(b+\Lambda^{\gamma} D_{s}+a_{11}+\Lambda^{\gamma} D_{r}\right) \lambda_{\gamma}+b \beta+b \beta b^{-\lambda_{\gamma} \tau}=0 .
$$


Lemma 1. If

$$
\begin{gathered}
3 \widehat{r}^{2}+\alpha>b, \\
2 b \beta>A_{1}^{2},
\end{gathered}
$$

and (6) holds, then we can get the following:

(1) When $\tau=0$, all characteristic roots of (12) have negative real parts

(2) When $\tau>0$, the characteristic equation (12) has a pair of purely imaginary roots $\pm i \omega^{*}$ at $\tau=\tau_{j}$, where

$$
\begin{aligned}
\omega^{* 2} & =2 b \beta-A_{1}^{2}, \\
A_{1} & =b+\Lambda^{\gamma} D_{s}+a_{11}+\Lambda^{\gamma} D_{r}, \\
\tau_{j} & =\frac{1}{\omega^{*}}\left(2 j \pi+\arccos \left(\frac{\omega^{* 2}-b \beta}{b \beta}\right)\right), \quad j=0,1,2 \ldots
\end{aligned}
$$

Proof. (1) When $\tau=0$, the characteristic equation (12) can be reduced to

$$
\lambda_{\gamma}^{2}-\lambda_{\gamma}\left[e+\Lambda^{\gamma} D_{s}-3 \widehat{r}^{2}+2(\alpha+1) \widehat{r}-a+\Lambda^{\gamma} D_{r}\right]+2 b \beta=0 .
$$

Considering (13), we can obtain that $b+\Lambda^{\gamma} D_{s}-3 \widehat{r}^{2}+$ $2(\alpha+1) \widehat{r}-\alpha+\Lambda^{\gamma} D_{r}<0$ and $2 b \beta>0$. Therefore, the characteristic equation (12) has a root with a negative real part (3). Suppose $\pm i \omega$ are a pair of pure imaginary roots of (12). Substituting $\pm i \omega$ into (12), we can get

$$
-\omega^{2}+i A_{1} \omega+b \beta+b \beta(\cos \omega \tau i \sin \omega \tau)=0,
$$

and through separating real and imaginary parts of the root, we can get

$$
\begin{aligned}
b \beta \cos \omega \tau+b \beta-\omega^{2} & =0, \\
A_{1} \omega \pm b \beta \sin \omega \tau & =0 .
\end{aligned}
$$

It is noticed that $\cos ^{2} \omega \tau+\sin ^{2} \omega \tau=1$, and we can get

$$
\omega^{2}\left(\omega^{2}+\left(A_{1}^{2}-2 b \beta\right)\right)=0 .
$$

Equation (13) has a unique positive real root $\omega^{* 2}$ if and only if (13) holds.

\section{Theorem 1.}

(1) When $\tau \in\left[0, \tau_{0}\right)$, system (3) is locally stable at the equilibrium $(\widehat{u}, \widehat{v})$ if (6) and (13) hold

(2) When $\tau \in\left[\tau_{0}, \infty\right)$, system (3) is unstable at the equilibrium $(\widehat{u}, \widehat{v})$ if (6) and (13) hold

(3) We can find the constant $\sigma>0$ and a smooth curve $\lambda(\tau):\left(\tau_{0}-\sigma, \tau_{0}+\sigma\right) \longrightarrow C$ when $\lambda\left(\tau_{0}\right)=i \omega^{*}$ and $\Delta\left(i \omega^{*}, \tau_{0}\right)=0$ for all $\tau \in\left(\tau_{0}-\sigma, \tau_{0}+\sigma\right)$

Proof. We refer to the Theorem 3.6 in the work of Ruan [25]. In consideration of that $\tau_{0}$ is the smallest value when the root of equation (12) has zero real part, when $t \in\left[0, \tau_{0}\right)$, the real parts of the characteristic roots are all negative. Through using G. J. Buther's Lemma [26], we know that $(\widehat{u}, \widehat{v})$ is locally stable when $\tau \in\left[0, \tau_{0}\right)$.

Now, we check the transversality condition

$$
\left.\frac{\mathrm{d}}{\mathrm{d} \tau} \operatorname{Re} \lambda(\tau)\right|_{\tau=\tau_{0}}>0
$$

Suppose $\lambda_{\gamma}=\mu+i \omega$ is the eigenvalue of (12), and by substituting $\lambda_{\gamma}$ into (12), we can obtain

$$
\begin{aligned}
\mu^{2}-\omega^{2}+A_{1} \mu+b \beta+b \beta b^{-\mu \tau} \cos \omega \tau & =0, \\
2 \mu \omega+A_{1} \omega-b \beta b^{-\mu \tau \sin \omega \tau} & =0 .
\end{aligned}
$$

Differentiating (20) with respect to $\tau$, we have

$$
\begin{aligned}
& \left(2 \mu+A_{1}-b \beta \tau b^{-\mu \tau} \cos \omega \tau\right) \frac{\mathrm{d} \mu}{\mathrm{d} \tau}-\left(2 \omega+b \beta \tau b^{-\mu \tau} \sin \omega \tau\right) \frac{\mathrm{d} \omega}{\mathrm{d} \tau}=b \beta b^{-\mu \tau}(\omega \sin \omega \tau+\mu \cos \omega \tau) \\
& \left(2 \omega+b \beta \tau b^{-\mu \tau} \sin \omega \tau\right) \frac{\mathrm{d} \mu}{\mathrm{d} \tau}+\left(2 \omega+A_{1}-A_{2} \tau b^{-\mu \tau} \cos \omega \tau\right) \frac{\mathrm{d} \omega}{\mathrm{d} \tau}=b \beta b^{-\mu \tau}(\omega \cos \omega \tau+\mu \sin \omega \tau)
\end{aligned}
$$

Eliminating the terms $(\mathrm{d} \omega / \mathrm{d} \tau)$ from the abovementioned equation, we get

$$
\begin{gathered}
{\left[\left(2 \mu+A_{1}-b \beta \tau e^{-\mu \tau} \cos \omega \tau\right)^{2}+\left(2 \omega+b \beta \tau e^{-\mu \tau} \sin \omega \tau\right)^{2}\right] \frac{\mathrm{d} \mu}{\mathrm{d} \tau}=} \\
b \beta b^{-\mu \tau}\left[(\omega \sin \omega \tau+\mu \cos \omega \tau)\left(2 \mu+A_{1}-b \beta \tau b^{-\mu \tau} \cos \omega \tau\right)\right] \\
+b \beta b^{-\mu \tau}\left[(\mu \sin \omega \tau+\omega \cos \omega \tau)\left(2 \omega+b \beta \tau b^{-\mu \tau} \sin \omega \tau\right)\right]
\end{gathered}
$$


To verify $(\mathrm{d} \mu / \mathrm{d} \tau)>0$, we just need to verify that

$$
(\omega \sin \omega \tau+\mu \cos \omega \tau)\left(2 \mu+A_{1}-b \beta \tau b^{-\mu \tau} \cos \omega \tau\right)+(\mu \sin \omega \tau+\omega \cos \omega \tau)\left(2 \omega+b \beta \tau b^{-\mu \tau} \sin \omega \tau\right)>0 .
$$

When $\tau=\tau_{0}, \mu=0, \omega=\omega^{*},(20)$ becomes

$$
\begin{aligned}
& b \beta \cos \omega^{*} \tau_{0}=\omega^{* 2}-b \beta, \\
& b \beta \sin \omega^{*} \tau_{0}=A_{1} \omega^{*} .
\end{aligned}
$$

By some simple calculations, we can get

$$
\omega^{* 4}+\left(A_{1}^{2}-2 b \beta\right) \omega^{* 2}=0
$$

So, (23) becomes

$$
\omega^{*} \sin \omega^{*} \tau_{0}\left(A_{1} \mp b \beta \tau_{0} \cos \omega^{*} \tau_{0}\right)+\omega^{*} \cos \omega^{*} \tau_{0}\left(2 \omega^{*} \pm b \beta \tau_{0} \sin \omega^{*} \tau_{0}\right)>0 .
$$

We must verify

$$
A_{1} \omega^{*} \sin \omega^{*} \tau_{0}+2 \omega^{*} \cos \omega^{*} \tau_{0}>0 .
$$

Substituting (25) into (27) yields

$$
\frac{1}{e b} A_{1}^{2} \omega^{* 2}-\frac{1}{e b}\left[2 \omega^{* 2}\left(A_{2}-\omega^{* 2}\right)\right]>0 \text {. }
$$

Since $b \beta>0$, we need only to verify that

$$
2 \omega^{* 4}+\left(A_{1}^{2}-2 b \beta\right) \omega^{* 2}=2 \omega^{* 4}-\omega^{* 4}=\omega^{* 4}>0 .
$$

The abovementioned inequality holds, so system (3) undergoes a Hopf bifurcation at $\tau=\tau_{0}$.
By applying the implicit function to $\Delta(\lambda, \tau)$, we can find the constant $\sigma>0$ and a smooth curve $\lambda(\tau):\left(\tau_{0}-\sigma, \tau_{0}+\right.$ $\sigma) \rightarrow C$ when $\lambda\left(\tau_{0}\right)=i \omega^{*}$ and $\Delta\left(i \omega^{*}, \tau_{0}\right)=0$ for all $\tau \in\left(\tau_{0}-\sigma, \tau_{0}+\sigma\right)$.

Now, by using proposition 6.5 in [28], we obtain that $(\widehat{u}, \widehat{v})$ is locally unstable for $\tau \in\left[\tau_{0}, \infty\right)$.

\section{The Stability of the Hopf Bifurcation}

As a matter of convenience, let $\tau=\tau_{0}+\nu$. Then, $\nu=0$ is the Hopf bifurcation value for system (3). Let $r_{i}-\widehat{r}=\bar{r}_{i}, s_{i}-\widehat{s}=\bar{s}_{i}$. For convenience, we leave off the bars of $\bar{r}_{i}$ and $\bar{s}_{i}$. System (3) can be rewritten as

$\left\{\dot{r}_{i}(t)=a_{11} r_{i}(t)-s_{i}-s_{i}(t-\tau)+(2(\alpha+1)-6 \widehat{r}) r_{i}^{2}(t)+D_{r} \sum_{j=1}^{n} L_{i j} r_{j}(t), \dot{s}_{i}(t)=b \beta r_{i}(t)-b s_{i}(t)+D_{s} \sum_{j=1}^{n} L_{i j} s_{j}(t)\right.$.

We rewrite the abovementioned equation as follows through using the orthogonality of the eigenvectors.

$$
\left\{\begin{array}{l}
\dot{r}_{i}(t)=a_{11} r_{i}(t)-s_{i}-s_{i}(t-\tau)+(2(\alpha+1)-6 \widehat{r}) r_{i}^{2}(t)+D_{r} \Lambda^{\alpha} r_{i}(t) \\
\dot{s}_{i}(t)=b \beta r_{i}(t)-b s_{i}(t)+D_{s} \Lambda^{\alpha} s_{i}(t)
\end{array}\right.
$$

We use $X(t)=\left(r_{i}(t), s_{i}(t)\right)^{T}$ to denote the solution of (31) and let $X_{t}(\theta)=X(t+\theta), \theta \in[-\tau, 0]$, where $T$ denotes the transpose, $X_{t} \in C\left([-\tau, 0], R^{2}\right) \triangleq C$. System (31) can become the functional differential equation as follows:

$$
\dot{X}_{t}=L_{v}\left(X_{t}\right)+R_{v}\left(X_{t}\right)
$$

where $L_{\nu}: C \rightarrow R^{2}, R_{\nu}: C \rightarrow R^{2}$; they have the following forms:

$$
\begin{aligned}
L_{\nu}(\phi) & =M_{0}\left(\begin{array}{l}
\phi_{1}(0) \\
\phi_{2}(0)
\end{array}\right)+M_{1}\left(\begin{array}{l}
\phi_{1}(-\tau) \\
\phi_{2}(-\tau)
\end{array}\right), \\
M_{0} & =\left(\begin{array}{cc}
a_{11}+D_{r} \Lambda^{\gamma} & -1 \\
b \beta & -b+D_{s} \Lambda^{\gamma}
\end{array}\right), \\
M_{1} & =\left(\begin{array}{cc}
0 & -1 \\
0 & 0
\end{array}\right), \\
R_{\nu}(\phi) & =\left(\begin{array}{c}
(-6 \hat{r}+2(\alpha+1)) \phi_{1}^{2}(0) \\
0
\end{array}\right) .
\end{aligned}
$$

We can let

$$
\eta(\theta, \nu)=M_{0} \delta(\theta)+M_{1} \delta(\theta+\tau),
$$

where $\delta(\cdot)$ is the Dirac function, and we can get 


$$
L_{\nu}(\phi)=\int_{-\tau}^{0} \phi(\theta) \mathrm{d} \eta(\theta, \nu), \quad \text { for } \phi \in C,
$$

where

$$
\mathrm{d} \eta(\theta, \nu)=M_{0} \delta(\theta) \mathrm{d} \theta+M_{1} \delta(\theta+\tau) \mathrm{d} \theta .
$$

Then, two operators $A_{v}$ and $F_{v}$ on $C^{1}\left([-\tau, 0], R^{2}\right) \triangleq C^{1}$ can be defined by

$$
\begin{aligned}
& \left(A_{\nu} \phi\right)(\theta)= \begin{cases}\frac{\mathrm{d} \phi}{\mathrm{d} \theta} & \theta \in[-\tau, 0), \\
\int_{-\tau}^{0} \phi(\xi) \mathrm{d} \eta(\xi, \nu), & \theta=0,\end{cases} \\
& \left(F_{\nu} \phi\right)(\theta)= \begin{cases}0, & \theta \in[-\tau, 0), \\
R_{\nu}(\phi), & \theta=0 .\end{cases}
\end{aligned}
$$

Then, (32) is transformed into

$$
\begin{aligned}
\dot{X}_{t} & =A_{\nu}\left(X_{t}\right)+F_{v}\left(X_{t}\right), \\
\left(A_{\nu}^{*} \psi\right)\left(\theta^{*}\right) & = \begin{cases}-\frac{\mathrm{d} \psi}{\mathrm{d} \theta^{*}}, & \theta^{*} \in(0, \tau], \\
\int_{-\tau}^{0} \psi(-\xi) \mathrm{d} \eta^{T}(\xi, \nu), & \theta^{*}=0,\end{cases}
\end{aligned}
$$

where $\psi \in C^{1}\left([0, \tau], R^{2}\right)$. We can define the bilinear form by taking the solution space of (38) as the complex space $C^{2}$.

$$
\left\langle\psi\left(\theta^{*}\right), \phi(\theta)\right\rangle=\bar{\psi}^{T}(0) \phi(0)-\int_{\theta=-\tau}^{0} \int_{\xi=0}^{\theta} \bar{\psi}^{T}(\xi-\theta) \mathrm{d} \eta(\theta) \phi(\xi) \mathrm{d} \xi,
$$

for $\quad \phi \in C\left([-\tau, 0], C^{2}\right), \psi \in C^{1}\left([0, \tau], C^{2}\right), \quad$ where $\eta(\theta)=\eta(\theta, 0)$.

From the proof in Lemma 1 , we can get that $\pm i \omega^{*}$ are eigenvalues of (12); thus, $\pm i \omega^{*}$ are the eigenvalues of $A_{0}$ and $A_{0}^{*}$, respectively. We set

$$
A_{0} q(\theta)=i \omega^{*} q(\theta), \quad \text { and } A_{0}^{*} q^{*}\left(\theta^{*}\right)=-i \omega^{*} q^{*}\left(\theta^{*}\right),
$$

and we know that $\left\langle q^{*}, q\right\rangle=1$ and $\left\langle q^{*}, \bar{q}\right\rangle=0$. Let

$$
q(\theta)=q(0) b^{i w^{*} \theta}=\left(\begin{array}{l}
q_{1} \\
q_{2}
\end{array}\right) b^{i w^{*} \theta}, \quad \text { and } q^{*}\left(\theta^{*}\right)=q^{*}(0) b^{i w^{*} \theta^{*}}=\left(\begin{array}{c}
q_{1}^{*} \\
q_{2}^{*}
\end{array}\right) b^{i w^{*} \theta^{*}}
$$

for $\theta \in[-\tau, 0], \theta^{*} \in(0, \tau)$.

So, we have

$$
q(\theta)=\left(\begin{array}{c}
1 \\
\frac{b \beta}{i \omega^{*}+b}
\end{array}\right) b^{i \omega^{*} \theta}, \quad \text { and } q^{*}\left(\theta^{*}\right)=\rho\left(\begin{array}{c}
\frac{b \beta}{-i \omega^{*}-a_{11}} \\
0
\end{array}\right) b^{i \omega^{*} \theta^{*}} .
$$

In order to ensure $\left\langle q^{*}(s), q(\theta)\right\rangle=1$, we should determine the value of $\rho$, and from (40), we get

$$
\begin{aligned}
\left\langle q^{*}(s), q(\theta)\right\rangle & =\bar{q}^{*}(0) q(0)-\int_{\theta=-\tau}^{0} \int_{\xi=0}^{\theta} \bar{q}^{*}(\xi-\theta) \mathrm{d} \eta(\theta) q(\xi) \mathrm{d} \xi \\
& =\bar{\rho}\left(\bar{q}_{1}^{*}\right)\left(q_{1}, q_{2}\right)^{T}-\int_{-\tau}^{0} \int_{0}^{\theta} \bar{\rho}\left(\bar{q}_{1}^{*}, \bar{q}_{2}^{*}\right) b^{-i \omega^{*}(\xi-\theta)} \mathrm{d} \eta(\theta)\left(q_{1}, q_{2}\right)^{T} b^{i \omega^{*} \xi} \mathrm{d} \xi \\
& =\bar{\rho}\left(\bar{q}_{1}^{*}+q_{2}+\tau_{0} b^{-i \omega^{*}} \tau_{0} a_{13} q_{2}\right), \\
& =\bar{\rho}\left(\bar{q}_{1}^{*}+q_{2}-\tau_{0} b^{-i \omega^{*}} \tau_{0} q_{2}\right) .
\end{aligned}
$$

Therefore, we can let

$$
\bar{\rho}=\frac{1}{\left(\bar{q}_{1}^{*}+q_{2}-\tau_{0} b^{-i \omega^{*} \tau_{0}} q_{2}\right)} .
$$

Then, we construct a coordinate for the center manifold $C_{0}$ at $v=0$ by using $q$ and $q^{*}$; the method is similar to that in [27]. Let $x_{t}=x_{t}(\theta)$ be a solution of (38), and we define

$$
\begin{aligned}
z(t) & \triangleq\left\langle q^{*}, x_{t}\right\rangle, \\
U(t, \theta) & \triangleq x_{t}(\theta)-2 \operatorname{Re}\{z(t) q(\theta)\} .
\end{aligned}
$$


We further get

$U(t, \theta)=U(z(t), \bar{z}(t), \theta)$

$$
\triangleq U_{20}(\theta) \frac{z^{2}}{2}+U_{11}(\theta) z \bar{z}+U_{02}(\theta) \frac{\bar{z}^{2}}{2}+U_{30}(\theta) \frac{z^{3}}{6}+\cdots,
$$

where $z$ and $\bar{z}$ are the local coordinates of the center manifold $C_{0}$ in directions $q$ and $q^{*}$, respectively.

We only take real solutions into account. For a solution $X(t) \in C_{0}$ of (26) with $\nu=0$, from (47), we have

$$
\begin{aligned}
\dot{z}(t) & =\left\langle q^{*}, \dot{x}_{t}\right\rangle, \\
& =i \omega^{*} z+\overline{q^{*}}(0) F_{0}(U(z, \bar{z}, \theta)+2 \operatorname{Re}\{z(t) q(\theta)\}), \\
& \triangleq i \omega^{*} z+\overline{q^{*}}(0) F_{0}(z, \bar{z}) .
\end{aligned}
$$

Thus, (49) can become

$$
\begin{gathered}
\dot{z}(t)=i \omega^{*} z+g(z, \bar{z}), \\
g(z, \bar{z})=\overline{q^{*}}(0) F_{0}(z, \bar{z}), \\
\triangleq \frac{g_{20}}{2} z^{2}+g_{11} z \bar{z}+\frac{g_{02}}{2} \bar{z}^{2}+\frac{g_{30}}{6} z^{3}+\frac{g_{21}}{2} z^{2} \bar{z} \\
+\frac{g_{12}}{2} z \bar{z}^{2}+\frac{g_{03}}{6} \bar{z}^{3}+\cdots
\end{gathered}
$$

Lemma 2. If we set

$$
k_{1}=-6 \widehat{u}+2(\alpha+1)
$$

then $g_{i j}$ can be explicitly expressed by (55).

Proof. Noticing (47) and (48), we obtain

$$
\begin{aligned}
x_{t}(\theta)= & U(t, \theta)+2 \operatorname{Re}\{z(t) q(\theta)\} \\
= & U_{20}(\theta) \frac{z^{2}}{2}+U_{11}(\theta) z \bar{z}+U_{02}(\theta) \frac{\bar{z}^{2}}{2}+z q+\bar{z} \bar{q}+\cdots, \\
= & U_{20}(\theta) \frac{z^{2}}{2}+U_{11}(\theta) z \bar{z}+U_{02}(\theta) \frac{\bar{z}^{2}}{2}+\left(1, q_{2}\right)^{T} b^{i \omega^{*} \theta} \\
& +\left(1, \bar{q}_{2}\right)^{T} e^{-i \omega^{*} \theta} \bar{z}+\cdots .
\end{aligned}
$$

In view of (52), substituting (53) into (51),

$$
\begin{aligned}
g(z, \bar{z}) & =\overline{q^{*}} F_{0}(z, \bar{z})=\overline{q^{*}}(0) F_{0}\left(x_{t}(\theta)\right), \\
& =\bar{\rho}\left(\bar{q}_{1}^{*}, 1\right)\left(\begin{array}{c}
K_{1} \varphi_{1}^{2}(0) \\
0
\end{array}\right),
\end{aligned}
$$

where $U_{20}^{(k)}, U_{11}^{(k)}$, and $U_{02}^{(k)}$ are the $k$-th components of $U_{20}, U_{11}$, and $U_{02}$, respectively, for $-\tau \leq \theta \leq 0$. The results in the theorem are valid.

Next, we will get the values of $U_{20}(\theta)$ and $U_{11}(\theta)$. From (38) and (47), we obtain

$$
\dot{U}=\dot{x}_{t}-\dot{z} q-\dot{\bar{z}} \bar{q},= \begin{cases}A_{0} U-2 \operatorname{Re}\left\{\bar{q}^{*}(0) F_{0} q(\theta)\right\}, & -\tau \leq \theta<0, \\ A_{0} U-2 \operatorname{Re}\left\{\bar{q}^{*}(0) F_{0} q(\theta)\right\}+F_{0}, & \theta=0,\end{cases}
$$

where

$$
K(z, \bar{z}, \theta)=K_{20} \frac{z^{2}}{2}+K_{11} z \bar{z}+K_{02} \frac{\bar{z}^{2}}{2}+\cdots
$$

From (48), we have

$$
\begin{aligned}
\dot{U}= & \dot{U}_{z} \dot{z}(t)+\dot{U}_{\bar{z}} \dot{\bar{z}}(t)=\left(U_{20}(\theta) z+U_{11}(\theta) \bar{z}+\cdots\right)\left(i \omega^{*} z(t)+g(z, \bar{z})\right) \\
& +\left(U_{11}(\theta) z+U_{02}(\theta) \bar{z}+\cdots\right)\left(-i \omega^{*} \bar{z}(t)+g(z, \bar{z})\right) .
\end{aligned}
$$


Substituting (48) into (56), we can get that

$$
\begin{aligned}
\dot{U} & =A_{0}\left(U_{20}(\theta) \frac{z^{2}}{2}+U_{11}(\theta) z \bar{z}+U_{02}(\theta) \frac{\bar{z}^{2}}{2}+\cdots\right)+K_{20}(\theta) \frac{z^{2}}{2}+K_{11}(\theta) z \bar{z}+K_{02}(\theta) \frac{\bar{z}^{2}}{2}+\cdots \\
& =\left(A_{0} U_{20}(\theta)+K_{20}(\theta)\right) \frac{z^{2}}{2}+\left(A_{0} U_{11}(\theta)+K_{11}(\theta) z \bar{z}+\left(A_{0} U_{02}(\theta)+K_{02}(\theta) \frac{\bar{z}^{2}}{2}\right)+\cdots\right.
\end{aligned}
$$

By comparing the coefficients of $z^{2}$ and $z \bar{z}$ from (58) and (59), we get

$$
\begin{aligned}
\left(A_{0}-2 i \omega^{*} I\right) U_{20}(\theta) & =-K_{20}(\theta), \\
A_{0} U_{11}(\theta) & =-K_{11}(\theta) .
\end{aligned}
$$

$$
\begin{aligned}
K(z, \bar{z}, \theta) & =-2 \operatorname{Re}\left\{\bar{q}^{*}(0) F_{0} q(\theta)\right\} \\
& =-\bar{q}^{*} F_{0} q(\theta)-q^{*}(0) \bar{F}_{0} \bar{q}(\theta) \\
& =-g(z, \bar{z}) q(\theta)-\bar{g}(z, \bar{z}) \bar{q}(\theta) \\
& =-\left(g_{20} \frac{z^{2}}{2}+g_{11} z \bar{z}+g_{02} \frac{\bar{z}^{2}}{2}+\cdots\right) q(\theta)-\left(\bar{g}_{20} \frac{\bar{z}^{2}}{2}+\bar{g}_{11} z \bar{z}+\bar{g}_{02} \frac{z^{2}}{2}+\cdots\right) \bar{q}(\theta) .
\end{aligned}
$$

By comparing the coefficients of $z^{2}$ and $z \bar{z}$ from (57) and (61), we get

$$
\begin{aligned}
& K_{20}(\theta)=-g_{20} q(\theta)-\bar{g}_{02} \bar{q}(\theta), \\
& K_{11}(\theta)=-g_{11} q(\theta)-\bar{g}_{11} \bar{q}(\theta) .
\end{aligned}
$$

Thus, we can get

$$
\dot{U}_{20}(\theta)=2 i \omega^{*} U_{20}(\theta)+g_{20} q(\theta)+\bar{g}_{02} \bar{q}(\theta) .
$$

Since $q(\theta)=\left(q_{1}, q_{2}\right)^{T} e^{i \omega^{*} \theta}$, we obtain

$$
U_{20}(\theta)=\frac{i g_{20}}{\omega^{*}} q(0) e^{i \omega^{*} \theta}+\frac{i \bar{g}_{02}}{\omega^{*}} \bar{q}(0) e^{-i \omega^{*} \theta}+G_{1} e^{2 i \omega^{*} \theta},
$$

where $G_{1}=\left(G_{1}^{(1)}, G_{1}^{(2)}\right)^{T}$ is a constant vector. We also can have

$$
U_{11}(\theta)=\frac{-i g_{11}}{\omega^{*}} q(0) e^{i \omega^{*} \theta}+\frac{i \bar{g}_{11}}{\omega^{*}} \bar{q}(0) e^{-i \omega^{*} \theta}+G_{2},
$$

where $G_{2}=\left(G_{2}^{(1)}, G_{2}^{(2)}\right)^{T}$ is a constant vector.
From the definition of $A_{0}$ and (60), we have

$$
\int_{-\tau}^{0} \mathrm{~d} \eta(\theta) U_{20}(\theta)=2 i \omega^{*} U_{20}(0)-K_{20}(0)
$$

and

$$
\int_{-\tau}^{0} \mathrm{~d} \eta(\theta) U_{11}(\theta)=-K_{11}(0),
$$

where $\eta(\theta)=\eta(\theta, 0)$. From (56), when $\theta=0$, we get

$$
\begin{aligned}
K(z, \bar{z}, 0) & =-2 \operatorname{Re}\left\{\bar{q}^{*}(0) F_{0} q(0)\right\}+F_{0}, \\
& =-g(z, \bar{z}) q(0)-\bar{g}(z, \bar{z}) \bar{q}(0)+F_{0} .
\end{aligned}
$$

Then, we obtain

$$
\begin{aligned}
K(z, \bar{z}, 0) & =-2 \operatorname{Re}\left\{\bar{q}^{*}(0) F_{0} q(0)\right\}+F_{0}, \\
& =-g(z, \bar{z}) q(0)-\bar{g}(z, \bar{z}) \bar{q}(0)+F_{0} .
\end{aligned}
$$

Therefore, we obtain

$$
K_{20} \frac{z^{2}}{2}+K_{11} z \bar{z}+K_{02} \frac{\bar{z}^{2}}{2}+\cdots=-\left(g_{20} \frac{z^{2}}{2}+g_{11} z \bar{z}+g_{02} \frac{\bar{z}^{2}}{2}+\cdots\right) q(0)-\left(\bar{g}_{20} \frac{\bar{z}^{2}}{2}+\bar{g}_{11} z \bar{z}+\bar{g}_{02} \frac{\bar{z}^{2}}{2}+\cdots\right) \bar{q}(0)+F_{0} .
$$


Comparing both sides of (70), we have

$$
\begin{aligned}
& K_{20}=-g_{20} q(0)-\bar{g}_{02} \bar{q}(0)+2\left(\begin{array}{c}
k_{1} \\
0
\end{array}\right), \\
& K_{11}=-g_{11} q(0)-\bar{g}_{11} \bar{q}(0)+2\left(\begin{array}{c}
2 k_{1} \\
0
\end{array}\right) .
\end{aligned}
$$

Since $i \omega^{*}$ is the eigenvalue of $A_{0}$ and $q(0)$ is the corresponding eigenvector, we get

$$
\begin{array}{r}
\left(i \omega^{*} I-\int_{-\tau}^{0} e^{i \omega^{*} \theta} \mathrm{d} \eta(\theta)\right) q(0)=0, \\
\left(-i \omega^{*} I-\int_{-\tau}^{0} e^{-i \omega^{*} \theta} \mathrm{d} \eta(\theta)\right) \bar{q}(0)=0 .
\end{array}
$$

Then, substituting (65) and (67) into (72), we obtain

$$
\left(2 i \omega^{*} I-\int_{-\tau}^{0} e^{2 i \omega^{*} \theta} \mathrm{d} \eta(\theta)\right) G_{1}=2\left(\begin{array}{c}
k_{1} \\
0
\end{array}\right),
$$

that is,

$$
K^{*} G_{1}=2\left(\begin{array}{c}
k_{1} \\
0
\end{array}\right)
$$

where

$$
K^{*}=\left(\begin{array}{cc}
2 i \omega *-a_{11}-D_{r} \Lambda^{\alpha} & e^{-2 i \omega^{*} \tau} \\
0 & 2 i \omega^{*}-D_{s} \Lambda^{\alpha}
\end{array}\right) .
$$

Thus,

$$
\begin{aligned}
G_{1}^{(1)} & =\frac{\left(\begin{array}{cc}
2 k_{1} & e^{-2 i \omega *} \tau \\
0 & 2 i \omega^{*}-D_{s} \Lambda^{\alpha}
\end{array}\right)}{\operatorname{Det}\left(K^{*}\right)}, \\
G_{1}^{(2)} & =\frac{\left(\begin{array}{cc}
2 i \omega^{*}-a_{11}-D_{s} \Lambda^{\alpha} & 2 k_{1} \\
0 & 0
\end{array}\right)}{\operatorname{Det}\left(K^{*}\right)} .
\end{aligned}
$$

Therefore, we can get the values of $U_{20}(\theta)$ and $U_{11}(\theta)$ from (64) and (65).

\section{Data Availability}

The data used to support the findings of this study are included within the article.

\section{Conflicts of Interest}

The authors declare that they have no conflicts of interest.

\section{Acknowledgments}

This work was supported by the Program for Innovative Research Team in Huainan Normal University (No. XJTD202008).

\section{References}

[1] J. G. Skellam, "Random dispersal in theoretical populations," Biometrika, vol. 38, no. 1-2, pp. 196-217, 1951.

[2] C. A. Klausmeier, "Regular and irregular patterns in semiarid vegetation," Science, vol. 284, no. 5421, pp. 1826-1828, 1999.

[3] H. Meinhardt, Models of Biological Pattern Formation, Academic Press, New York, NY, USA, 1982.

[4] J. A. Sherratt, "An analysis of vegetation stripe formation in semi-arid landscapes," Journal of Mathematical Biology, vol. 51, no. 2, pp. 183-197, 2005.

[5] J.-F. Zhang, W.-T. Li, and X.-P. Yan, "Hopf bifurcation and Turing instability in spatial homogeneous and inhomogeneous predator-prey models," Applied Mathematics and Computation, vol. 218, no. 5, pp. 1883-1893, 2011.

[6] R. C. Wu, Y. Shao, and L. P. Chen, "Turing and Hopf Bifurcation of Gierer-Meinhardt Activator-Substrate Model," Electronic Journal of Differential Equations, vol. 2017, no. 173, pp. 1-19, 2017.

[7] F. Borgogno, P. Dodorico, F. Laio, and L. Ridolfi, "Mathematical models of vegetation pattern formation in ecohydrology," Reviews of Geophysics, vol. 47, pp. 1-36, 2009.

[8] A. M. Turing, "The chemical basis of morphogenesis," Philosophical Transactions of the Royal Society of London. Series B, Biological Sciences, vol. 237, no. 641, pp. 37-72, 1952.

[9] M. Banerjee and S. Petrovskii, "Self-organised spatial patterns and chaos in a ratio-dependent predator-prey system," Theoretical Ecology, vol. 4, no. 1, pp. 37-53, 2011.

[10] H.-B. Shi, S. Ruan, Y. Su, and J.-F. Zhang, "Spatiotemporal dynamics of a diffusive Leslie-Gower predator-prey model with ratio-dependent functional response," International Journal of Bifurcation and Chaos, vol. 25, no. 5, Article ID 1530014, 2015.

[11] A. H. Abbasian, H. Fallah, and M. R. Razvan, "Symmetric bursting behaviors in the generalized FitzHugh-Nagumo model," Biological Cybernetics, vol. 107, no. 4, pp. 465-476, 2013.

[12] M. Ringquist, "On dynamical behavior of FitzHugh-Nagumo systems," Research Reports in Mathematics, vol. 5, 2006, https://www2.math.su.se/reports/2006/5.

[13] R. FitzHugh, "Impulses and physiological states in theoretical models of nerve membrane," Biophysical Journal, vol. 1, no. 6, pp. 445-466, 1961.

[14] M. Perc, "Spatial coherence resonance in excitable media," Physical Review Journals E, vol. 72, 2005.

[15] M. Perc, "Persistency of noise-induced spatial periodicity in excitable media," Europhysics Letters (EPL), vol. 72, no. 5, pp. 712-718, 2005.

[16] M. Perc, "Spatial decoherence induced by small-world connectivity in excitable media," New Journal of Physics, vol. 7, pp. 252-261, 2005.

[17] M. Perc, "Effects of small-world connectivity on noise-induced temporal and spatial order in neural media," Chaos, Solitons \& Fractals, vol. 31, no. 2, pp. 280-291, 2007.

[18] M. Perc, "Fluctuating excitability: a mechanism for self-sustained information flow in excitable arrays," Chaos, Solitons \& Fractals, vol. 32, no. 3, pp. 1118-1124, 2007.

[19] G. S. Medvedev and N. Kopell, "Synchronization and transient dynamics in the chains of electrically coupled Fitzhugh-nagumo oscillators," SIAM Journal on Applied Mathematics, vol. 61, no. 5, pp. 1762-1801, 2001.

[20] T. Kostova, R. Ravindran, and M. Schonbek, "FitzhughNagumo revisited: types of bifurcations, periodical forcing and stability regions by a Lyapunov functional," International 
Journal of Bifurcation and Chaos, vol. 14, no. 3, pp. 913-925, 2004.

[21] C. J. Xu and P. L. Li, "Bifurcations for a FitzHugh-Nagumo model with time delays," International Journal of Mathematical, vol. 7, no. 1, pp. 103-107, 2013, https://zenodo.org/ record/1335742.

[22] M. Ringkvist and Y. Zhou, "On the dynamical behaviour of FitzHugh-Nagumo systems: revisited," Nonlinear Analysis: Theory, Methods \& Applications, vol. 71, no. 7-8, pp. 26672687, 2009.

[23] A. Berman and R. J. Plemmons, Nonnegative Matrices in the Mathematical Sciences, Academic Press, New York, NY, USA, 1979.

[24] J. Petit, M. Asllani, D. Fanelli, B. Lauwens, and T. Carletti, "Pattern formation in a two-component reaction-diffusion system with delayed processes on a network," Physica A: Statistical Mechanics and Its Applications, vol. 462, pp. 230249, 2016.

[25] S. Ruan, "Absolute stability, conditional stability and bifurcation in Kolmogorov-type predator-prey systems with discrete delays," Quarterly of Applied Mathematics, vol. 59, no. 1, pp. 159-173, 2001.

[26] H. L. Freedman and V. Sree Hari Rao, "The trade-off between mutual interference and time lags in predator-prey systems," Bulletin of Mathematical Biology, vol. 45, no. 6, pp. 991-1004, 1983.

[27] B. Hassard, N. Kazarinoff, and Y. Wan, Theory and Application of Hopf Bifurcation, Cambridge University Press, Cambridge, UK, 1981. 